\title{
A REFINEMENT OF PELLET'S THEOREM
}

\section{MORRIS MARDEN}

1. Introduction. S. Lipka ${ }^{1}$ has recently announced a refinement of the classic theorem of Cauchy that all the zeros of the polynomial

$$
f(z)=a_{0}+a_{1} z+\cdots+a_{n} z^{n}, \quad a_{n} \neq 0,
$$

lie in the circle $|z| \leqq r$, where $r$ is the positive root of the real equation

$$
F_{n}(z)=\left|a_{0}\right|+\left|a_{1}\right| z+\cdots+\left|a_{n-1}\right| z^{n-1}-\left|a_{n}\right| z^{n}=0 .
$$

Lipka's refinement consists in replacing the circle $|z|=r$ by a curve $G\left(r_{0}, r ; n, \alpha_{0}\right)$ which bounds a gear-wheel region. This region is formed by deleting from the circle $|z| \leqq r$ the points common to the annular ring $0<r_{0}<|z| \leqq r$ and to the $n$ sectors

$$
\frac{\alpha_{0}}{n}-\frac{\pi}{2 n}+\frac{2 \pi_{k}}{n} \leqq \arg z \leqq \frac{\alpha_{0}}{n}+\frac{\pi}{2 n}+\frac{2 \pi k}{n},
$$

$k=0,1, \cdots, n-1$. In these formulas $r_{0}$ is the positive root of the equation

$$
\Phi_{n}(z)=\left|a_{1}\right|+\left|a_{2}\right| z+\cdots+\left|a_{n-1}\right| z^{n-2}-\left|a_{n}\right| z^{n-1}=0
$$

and $\alpha_{0}=\arg a_{0} / a_{n}$.

Now, the Cauchy theorem is but a special case of the following theorem due to Pellet. ${ }^{2}$

Pellet's Theorem. If the polynomial

$$
f(z)=a_{0}+a_{1} z+\cdots+a_{p} z^{p}+\cdots+a_{n} z^{n}, \quad a_{p} \neq 0,
$$

is such that the real polynomial

$$
\begin{aligned}
F_{p}(z)= & \left|a_{0}\right|+\left|a_{1}\right| z+\cdots+\left|a_{p-1}\right| z^{p-1}-\left|a_{p}\right| z^{p} \\
& +\left|a_{p+1}\right| z^{p+1}+\cdots+\left|a_{n}\right| z^{n}
\end{aligned}
$$
1947.

Presented to the Society, September 3, 1947; received by the editors August 22,

${ }^{1}$ S. Lipka, Monatshefte für Mathematik und Physik vol. 50 (1944) pp. 209-221.

2 A. Pellet, Bull. Sci. Math. vol. 5 (1881) pp. 393-395. The converse to this theorem was discussed by J. L. Walsh, Ann. of Math. vol. 26 (1924-1925) pp. 59-64 and A. Ostrowski, Bull. Amer. Math. Soc. vol. 47 (1941) pp. 742-746. See also M. Marden, The geometry of the zeros of a polynomial in a complex variable, chap. 7 , to be published as a volume of Mathematical Surveys. 
has two positive zeros $r$ and $R$ with $r<R$, then $f(z)$ has exactly $p$ zeros in or on the circle $|z| \leqq r$ and no zeros in the annular ring $r<|z|<R$.

It is Pellet's theorem which we propose to refine as indicated in the following theorem.

THEOREM 1.1. Under the hypotheses of Pellet's Theorem the polynomial

$$
\begin{aligned}
\Phi_{p}(z)= & \left|a_{1}\right|+\left|a_{2}\right| z+\cdots+\left|a_{p-1}\right| z^{p-2}-\left|a_{p}\right| z^{p-1} \\
& +\left|a_{p+1}\right| z^{p}+\cdots+\left|a_{n}\right| z^{n-1}
\end{aligned}
$$

has also two positive zeros $r_{0}$ and $R_{0}$ with

$$
r_{0}<r<R<R_{0} \text {. }
$$

Furthermore, $f(z)$ has exactly $p$ zeros in or on the curve $G\left(r_{0}, r ; p, \alpha_{0}\right)$ where $\alpha_{0}=\arg a_{0} / a_{p}$ and no zeros between the curves $G\left(r_{0}, r ; p, \alpha_{0}\right)$ and $G\left(R, R_{0} ; p, \alpha_{0}+\pi\right)$.

Theorem 1.1 will be proved in $\$ 2$ and applied in $\$ 3$ to the refinement of various known bounds on the zeros of a polynomial. Finally, the theorem will be generalized in $\$ 4$, first by replacing the polynomial $\Phi_{p}(z)$ by the polynomial $\Phi_{k p}(z)=F_{p}(z)-\left|a_{k}\right| z^{k}$ and secondly by replacing the polynomial $f(z)$ by a power series.

2. Proof of Theorem 1.1. Let us first prove the existence of the roots $r_{0}$ and $R_{0}$ of equation $\Phi_{p}(z)=0$ and the validity of inequality (1.8). Since $r$ and $R$ are the positive zeros of $F_{p}(z)$, it follows from (1.6) that, for any sufficiently small positive number $\epsilon$,

$$
F_{p}(\rho)<0 \quad \text { if } r+\epsilon \leqq \rho \leqq R-\epsilon .
$$

In view of the equation

$$
F_{p}(z)=\left|a_{0}\right|+z \Phi_{p}(z),
$$

the zeros $r$ and $R$ of $F_{p}(z)$ satisfy the relations

$$
\Phi_{p}(r)=-\left|a_{0}\right| / r<0, \quad \Phi_{p}(R)=-\left|a_{0}\right| / R<0 .
$$

When taken together with the facts that

$$
\Phi_{p}(0)>0, \quad \Phi_{p}(\infty)>0,
$$

the relations (2.3) imply the existence of two positive zeros $r_{0}$ and $R_{0}$ of $\Phi_{p}(z)$ and the validity of inequality (1.8), as well as the inequality

$$
\Phi_{p}(\rho)<0
$$

for $r_{0}+\epsilon \leqq \rho \leqq R_{0}-\epsilon$. 
Let us now set $z=\rho e^{i \theta}$ and

$$
a_{k} / a_{p}=A_{k} e^{\alpha_{k} i}, \quad k=0,1, \cdots, n \text {. }
$$

In this notation, the real part of $\rho^{p} f(z) / a_{p} z^{p}$ is

$$
\begin{aligned}
\operatorname{Re}\left[\rho^{p} f(z) / a_{p} z^{p}\right]= & \sum_{j=0}^{p-1} A_{j} \rho^{j} \cos \left[(p-j) \theta-\alpha_{j}\right]+\rho^{p} \\
& +\sum_{j=p+1}^{n} A_{j} \rho^{j} \cos \left[(j-p) \theta+\alpha_{j}\right]
\end{aligned}
$$

and the inequalities (2.1) and (2.5) become

(2.8) $\rho^{p}>A_{0}+A_{1} \rho+\cdots+A_{p-1} \rho^{p-1}+A_{p+1} \rho^{p+1}+\cdots+A_{n} \rho^{n}$

for $r+\epsilon \leqq \rho \leqq R-\epsilon$, and

(2.9) $\rho^{p}>A_{1} \rho+A_{2} \rho^{2}+\cdots+A_{p-1} \rho^{p-1}+A_{p+1} \rho^{p+1}+\cdots+A_{n} \rho^{n}$

for $r_{0}+\epsilon \leqq \rho \leqq R_{0}-\epsilon$.

On substituting from inequality (2.8) into (2.7), we find

$$
\begin{aligned}
\operatorname{Re}\left(\rho^{p} f(z) / a_{p} z^{p}\right)> & \sum_{j=0}^{p-1} A_{j} \rho^{i}\left\{\cos \left[(p-j) \theta-\alpha_{j}\right]+1\right\} \\
& +\sum_{j=p-1}^{n} A_{j} \rho^{j}\left\{\cos \left[(j-p) \theta+\alpha_{j}\right]+1\right\} \geqq 0
\end{aligned}
$$

for $r+\epsilon \leqq \rho \leqq R-\epsilon$. On substituting from inequality (2.9) into (2.7), we find

$$
\begin{aligned}
\operatorname{Re}\left(\rho^{p} f(z) / a_{p} z^{p}\right)> & A_{0} \cos \left(p \theta-\alpha_{0}\right) \\
& +\sum_{j=1}^{p-1} A_{j} \rho^{j}\left\{\cos \left[(p-j) \theta-\alpha_{j}\right]+1\right\} \\
& +\sum_{j=p-1}^{n} A_{j} \rho^{j}\left\{\cos \left[(j-p) \theta+\alpha_{j}\right]+1\right\}
\end{aligned}
$$

for $r_{0}+\epsilon \leqq \rho \leqq R_{0}-\epsilon$. The right side of (2.11) is surely non-negative if $\theta$ is such that $\cos \left(p \theta-\alpha_{0}\right) \geqq 0$, that is, such that

$$
-\frac{\pi}{2}+2 \pi k \leqq p \theta-\alpha_{0} \leqq \frac{\pi}{2}+2 \pi k,
$$

where $k$ is an integer; that is, if

$$
\frac{\alpha_{0}}{p}-\frac{\pi}{2 p}+\frac{2 \pi k}{p} \leqq \theta \leqq \frac{\alpha_{0}}{p}+\frac{\pi}{2 p}+\frac{2 \pi k}{p}, \quad k=0,1, \cdots, p .
$$


In other words,

$$
\operatorname{Re}\left(\rho^{p} f(z) / a_{p} z^{p}\right)>0
$$

and hence $f(z) \neq 0$ at all points $z$ between the curves $G\left(r_{0}, r ; p, \alpha_{0}\right)$ and $G\left(R, R_{0} ; p, \alpha_{0}+\pi\right)$.

Inequality (2.12) also may be used to show that in or on the curve $G\left(r_{0}, r ; p, \alpha_{0}\right)$, there are exactly $p$ zeros of $f(z)$. For, let us consider the net change $\Delta_{G_{\epsilon}}$ arg $w$ in the argument of the point $w=\left[\rho^{p} f(z) / a_{p} z^{p}\right]$ as $z$ describes counterclockwise the curve $G_{\epsilon}=G\left(r_{0}+\epsilon, r+\epsilon ; p, \alpha_{0}\right)$ where $\epsilon$ is a small positive number. Since $\operatorname{Re}(w)>0, w$ describes a closed curve entirely in the right-half $w$-plane. That is, $\Delta_{G_{e}}$ arg $w=0$ on this curve. This means that the function $w$ has as many zeros as poles in the curve $G_{\epsilon}$ and this, in turn, means that $f(z)$ has precisely $p$ zeros in $G_{\epsilon}$ for every sufficiently small positive $\epsilon$.

3. Applications. Let us first apply Theorem 1.1 to the class of polynomials

$$
\begin{aligned}
f(z)= & b_{0} e^{i \beta_{0}}+\left(b_{1}-b_{0}\right) e^{i \beta_{1} z}+\cdots \\
& +\left(b_{m}-b_{m-1}\right) e^{i \beta_{m}} z^{m}-b_{m} e^{i \beta_{m+1}} z^{m+1}
\end{aligned}
$$

where the $b_{j}$ are real numbers such that

$$
b_{p-1}<b_{p-2}<\cdots<b_{0}<0<b_{m}<b_{m-1}<\cdots<b_{p} .
$$

The corresponding polynomials $F_{p}(z)$ and $\Phi_{p}(z)$ are

$$
\begin{aligned}
F_{p}(z)= & -b_{0}+\left(b_{0}-b_{1}\right) z+\cdots+\left(b_{p-2}-b_{p-1}\right) z^{p-1} \\
& -\left(b_{p}-b_{p-1}\right) z^{p}+\left(b_{p}-b_{p+1}\right) z^{p+1}+\cdots \\
& +\left(b_{m-1}-b_{m}\right) z^{m}+b_{m} z^{m+1} \\
\Phi_{p}(z)= & \left(b_{0}-b_{1}\right)+\cdots+\left(b_{p-2}-b_{p-1}\right) z^{p-2} \\
& -\left(b_{p}-b_{p-1}\right) z^{p-1}+\left(b_{p}-b_{p+1}\right) z^{p}+\cdots \\
& +\left(b_{m-1}-b_{m}\right) z^{m-1}+b_{m} z^{m}
\end{aligned}
$$

On defining

$$
g(z)=b_{0}+b_{1} z+\cdots+b_{m} z^{m}
$$

we may write

$$
F_{p}(z)=(z-1) g(z), \quad z \Phi_{p}(z)=b_{0}+g(z)(z-1) .
$$

Clearly $F_{p}(1)=0$. Since $F_{p}(1+\delta)=\delta g(1+\delta)$, then for $\delta$ sufficiently small $g(1)>0$ implies that $F_{p}(1+\delta)>0$ or $<0$ according as $\delta>0$ or $<0$ and $g(1)<0$ implies that $F_{p}(1+\delta)<0$ or $>0$ according as $\delta>0$ or 
$<0$. That is, using the notation of Theorem 1.1 , we see that

$$
\begin{aligned}
r_{0}<r<1 & =R<R_{0} & & \text { if } g(1)>0, \\
r_{0}<r & =1<R<R_{0} & & \text { if } g(1)<0, \\
\alpha_{0} & =\beta_{0}-\beta_{p}+\pi . & &
\end{aligned}
$$

We thereby conclude that the following is true.

TheOREM 3.1. Let $f(z), \Phi_{p}(z)$ and $g(z)$ denote the polynomials (3.1), (3.4) and (3.5) respectively. Then, if $g(1)>0, f(z)$ has exactly $p$ zeros in the curve $G\left(r_{0}, 1 ; p, \beta_{0}-\beta_{p}+\pi\right)$ and $g(z)$ has $p$ zeros in the curve $G\left(r_{0}, 1 ; p, \pi\right)$. If $g(1)<0, f(z)$ has $p$ zeros in or on the curve $G\left(r_{0}, 1\right.$; $\left.p, \beta_{0}-\beta_{p}+\pi\right)$ and $g(z)$ has $p-1$ zeros in or on the curve $G\left(r_{0}, 1 ; p, \pi\right)$.

An analogous result for $g(z)$ with, however, curve $G\left(r_{0}, 1 ; p, \pi\right)$ replaced by the circle $|z|=1$ was first stated by Berwald. ${ }^{3}$ His result was a generalization of the Kakeya-Eneström ${ }^{4}$ theorem that all the zeros of the real polynomial (3.5) with $0<b_{0}<b_{1}<\cdots<b_{n}$ lie in or on the unit circle $|z|=1$. Our analogy to the Kakeya-Eneström theorem will be included in the following theorem.

THEOREM 3.2. Every polynomial of the form

$$
f(z)=\sum_{j=0}^{n}\left(b_{j}-b_{j-1}\right) e^{i \beta_{j} z^{j}}, \quad b_{-1}=b_{n}=0<b_{0}<b_{1}<\cdots<b_{n-1},
$$

has all of its zeros in or on the curve $G\left(r_{0}, 1 ; n, \beta_{0}-\beta_{n}+\pi\right)$ where $r_{0}$ is the positive root of the equation

$\Phi_{n}=\left(b_{1}-b_{0}\right)+\left(b_{2}-b_{1}\right) z+\cdots+\left(b_{n-1}-b_{n-2}\right) z^{n-2}-b_{n-1} z^{n-1}=0$.

Furthermore, every polynomial of the form

$$
g(z)=b_{0}+b_{1} z+\cdots+b_{n-1} z^{n-1}, \quad 0<b_{0}<b_{1}<\cdots<b_{n-1},
$$

has all of its zeros in or on the curve $G\left(r_{0}, 1 ; n, \pi\right)$.

This theorem may be derived from Theorem 3.1 indirectly by a limiting process or directly by the same methods as used for Theorem 3.1.

In our next application, we shall use Theorem 1.1 just in the case $p=n$. This restriction is made only to simplify the statement of results, since a similar application may be made when $p$ is an arbitrary integer, $0<p \leqq n$. The result to be proved is the following.

${ }^{3}$ L. Berwald, Math. Zeit. vol. 37 (1933) pp. 61-76.

4. Kakeya, Tôhoku Math. J. vol. 2 (1912) pp. 140-142 and G. Eneström, Ibid. vol. 18 (1920) pp. 34-36. 
THEOREM 3.3. Let $\lambda_{1}, \lambda_{2}, \cdots, \lambda_{n}$ and $\mu_{1}, \mu_{2}, \cdots, \mu_{n-1}$ be any two sets of numbers such that

$\sum_{j=1}^{n}\left(1 / \lambda_{j}\right)=1, \quad \sum_{j=1}^{n-1}\left(1 / \mu_{j}\right)=1 ; \quad 0<\mu_{j} \leqq \lambda_{j}, j=1,2, \cdots, n-1$.

For the polynomial $f(z)=a_{0}+a_{1} z+\cdots+a_{n} z^{n}$, let

$$
\begin{aligned}
M & =\max \left[\lambda_{k}\left|a_{n-k}\right| /\left|a_{n}\right|\right]^{1 / k}, \quad k=1,2, \cdots, n, \\
M_{0} & =\max \left[\mu_{k}\left|a_{n-k}\right| /\left|a_{n}\right|\right]^{1 / k}, \quad k=1,2, \cdots, n-1 .
\end{aligned}
$$

Then all the zeros of $f(z)$ lie in or on the curve $G\left(M_{0}, M ; n, \alpha_{0}\right)$, where $\alpha_{0}=\arg \left(a_{0} / a_{n}\right)$.

From (3.6) and (3.7), obviously $0<M_{0}<M$. Also,

$$
\lambda_{k}\left|a_{n-k}\right| \leqq\left|a_{n}\right| M^{k}, \quad \mu_{k}\left|a_{n-k}\right| \leqq\left|a_{n}\right| M_{0}^{k}
$$

and thus

$$
\begin{aligned}
& \sum_{k=1}^{n}\left|a_{n-k}\right| M^{n-k} \leqq \sum_{k=1}^{n}\left(1 / \lambda_{k}\right)\left|a_{n}\right| M^{n}=\left|a_{n}\right| M^{n}, \\
& \sum_{k=1}^{n-1}\left|a_{n-k}\right| M_{0}^{n-k} \leqq \sum_{k=1}^{n-1}\left(1 / \mu_{k}\right)\left|a_{n}\right| M_{0}^{n}=\left|a_{n}\right| M_{0}^{n} .
\end{aligned}
$$

An equality in (3.8) would imply that $M$ is the positive root $r$ of the equation (1.2) whereas an inequality in (3.8) would imply that $M>r$. Likewise, an equality in (3.9) would imply that $M_{0}$ is the positive root $r_{0}$ of the equation (1.4) whereas an inequality in (3.9) would imply that $M_{0}>r_{0}$. Since by Theorem 1.1 all the zeros of $f(z)$ lie in or on the curve $G\left(r_{0}, r ; n, \alpha_{0}\right)$, they surely all lie in or on the curve $G\left(M_{0}, M ; n, \alpha_{0}\right)$.

Theorem 3.3 whose proof we have just completed is a refinement of the result due to Fujiwara ${ }^{5}$ that all the zeros of $f(z)$ lie in or on the circle $|z| \leqq M$.

As a simple application of Theorem 3.3, let us take $\lambda_{j}=n$ for $j=1,2, \cdots, n$ and $\mu_{j}=n-1$ for $j=1,2, \cdots, n-1$. We obtain thereby the following corollary.

Corollary 3.3a. For the polynomial $f(z)=a_{0}+a_{1} z+\cdots+a_{n} z^{n}$ let $N=\max \left[n\left|a_{n-k} / a_{n}\right|\right]^{1 / k}, k=1,2, \cdots, n$, and $N_{0}=\max [(n-1)$ $\left.\left|a_{n-k} / a_{n}\right|\right]^{1 / k}, k=1,2, \cdots, n-1$. Then all the zeros of $f(z)$ lie in or on the curve $G\left(N_{0}, N ; n, \alpha_{0}\right)$ where $\alpha_{0}=\arg \left(a_{0} / a_{n}\right)$.

5 M. Fujiwara. Tôhoku Math. J. vol. 10 (1916) pp. 167-171. 
As another simple application of Theorem 3.3, let us take

$$
\begin{aligned}
\lambda_{k} & =\sum_{j=0}^{n-1}\left|a_{j}\right| /\left|a_{n-k}\right|, & k=0,1,2,3, \cdots, n, \\
\mu_{k} & =\sum_{j=1}^{n-1}\left|a_{j}\right| /\left|a_{n-k}\right|, & k=0,1,2, \cdots, n-1 .
\end{aligned}
$$

Clearly,

$$
\sum_{j=1}^{n} 1 / \lambda_{k}=1, \quad \sum_{j=1}^{n-1} 1 / \mu_{j}=1
$$

Here

$$
M=\max \left[\sum_{j=0}^{n-1}\left|a_{j}\right| /\left|a_{n}\right|\right]^{1 / k}=\lambda_{0} \quad \text { or } \quad \lambda_{0}^{1 / n}
$$

according as $\lambda_{0}>1$ or $<1$, and

$$
M_{0}=\max \left[\sum_{j=1}^{n-1}\left|a_{i}\right| /\left|a_{n}\right|\right]^{1 / k}=\lambda_{0} \text { or } \mu_{0}^{1 / n}
$$

according as $\mu_{0}>1$ or $<1$. We thereby obtain the following corollary.

COROLlaRY 3.3b. For the polynomial $f(z)=a_{0}+a_{1} z+\cdots+a_{n} z^{n}$, let

$$
\lambda_{0}=\sum_{j=0}^{n-1}\left|a_{j}\right| /\left|a_{n}\right| \quad \text { and } \quad \mu_{0}=\sum_{j=1}^{n-1}\left|a_{i}\right| /\left|a_{n}\right| .
$$

Let $\gamma=\lambda_{0}$ or $\lambda_{0}^{1 / n}$ according as $\lambda_{0}>1$ or $\left\langle 1\right.$, and let $\delta=\mu_{0}$ or $\mu_{0}^{1 / n}$ according as $\mu_{0}>1$ or $<1$. Then all the zeros of $f(z)$ lie in or on the curve $G\left(\delta, \gamma ; n, \alpha_{0}\right)$ where $\alpha_{0}=\arg a_{0} / a_{n}$.

4. Generalizations. Let us define $\Psi_{k p}(z)=F_{p}(z)-\left|a_{k}\right| z^{k}, k \neq p$. Since $\Psi_{0 p}(z)=z \Phi_{p}(z)$, the positive zeros of $\Phi_{p}(z)$ are also the positive zeros of $\Psi_{0 p}(z)$. By modifying somewhat the details of proof of Theorem 1.1, we may prove the following generalization.

TheOREM 4.1. Under the hypotheses of Pellet's Theorem the polynomial

$$
\Psi_{k p}(z)=F_{p}(z)-\left|a_{k}\right| z^{k}, \quad k \neq p, a_{k} \neq 0,
$$

has also two positive zeros $r_{k}$ and $R_{k}$ with $r_{k}<r<R<R_{k}$. Furthermore $f(z)$ has exactly $p$ zeros in or on the curve $G\left(r_{k}, r ; p-k, \alpha_{k}\right)$ where $\alpha_{k}=\arg \left(a_{k} / a_{p}\right)$ and none between the curves $G\left(r_{k}, r ; p-k, \alpha_{k}\right)$ and $G\left(R, R_{k} ; p-k, \alpha_{k}+\pi\right)$. 
Our final generalization will consist in replacing the polynomial $f(z)$ of Theorem 4.1 by a power series.

THEOREM 4.2. If the power series

$$
f(z)=a_{0}+a_{1} z+\cdots+a_{p} z^{p}+\cdots, \quad a_{k} a_{p} \neq 0,
$$

having a radius of convergence of $\rho, 0<\rho \leqq \infty$, is such that each polynomial

$$
\begin{aligned}
F_{n p}(z)= & \left|a_{0}\right|+\left|a_{1}\right| z+\cdots+\left|a_{p-1}\right| z^{p-1}-\left|a_{p}\right| z^{p} \\
& +\left|a_{p+1}\right| z^{p+1}+\cdots+\left|a_{n}\right| z^{n}
\end{aligned}
$$

with $n \geqq N>p$ has a positive zero $r^{(n)}, r^{(n)} \leqq \rho_{1}<\rho$, then the function $F_{p}(z)=\lim _{n=\infty} F_{n p}(z)$ has a positive zero $r<\rho$; the function

$$
\Psi_{k p}(z)=F_{p}(z)-\left|a_{k}\right| z^{k}, \quad k \neq p,
$$

has a positive zero $r_{k}, r_{k}<r<\rho$, and the function $f(z)$ has exactly $p$ zeros in or on the curve $G\left(r_{k}, r ; p-k, \alpha_{k}\right)$ and hence in the curve $G\left(r_{k}, \rho\right.$; $\left.p-k, \alpha_{k}\right)$.

This theorem results from Theorem 4.1 on the use of the Hurwitz theorem that within its circle of convergence a non-constant power series $f(z)=\sum_{j=0}^{\infty} a_{j} z^{i}$ has as zeros the limit points of the zeros of the polynomials $f_{n}(z)=\sum_{j=0}^{n} a_{j} z^{i}$.

If $F_{n p}(z)$ has two positive zeros in $|z|<\rho$, we may choose $r^{(n)}$ as the smaller one. Letting

$$
\Psi_{n ` p}(z)=F_{n p}(z)-\left|a_{k}\right| z^{k},
$$

we see that $\Psi_{n k p}(z)$ has a positive zero $r_{k}^{(n)}, r_{k}^{(n)}<r^{(n)}$. Clearly, the power series $F_{p}(z)$ and $\Psi_{k p}(z)$ have the same radius $\rho$ of convergence and have respectively the positive zeros $r=\lim _{n=\infty} r^{(n)}$ and $r_{k}=\lim _{n=\infty} r_{k}^{(n)}$, with $r_{k}<r<\rho$. Now, given any small positive $\epsilon$, we can find an $N>0$ such that the circle of radius $\epsilon$ drawn about the point $z=r$ will contain $r^{(n)}$ for all $n \geqq N$ and the circle of radius $\epsilon$ drawn about $z=r_{k}$ will contain $r_{k}^{(n)}$ for all $n \geqq N$. This means that in or on the curve $G\left(r_{k}+\epsilon, r+\epsilon ; p-k, \alpha_{k}\right)$, which for any sufficiently small positive $\epsilon$ is contained in the circle $|z|<\rho$, lie exactly $p$ zeros of each polynomial $f_{n}(z)$ for all $n \geqq N$. Since a circle of radius $\epsilon$ about any zero of $f(z)$ in $|z|<\rho$ contains a zero of each $f_{n}(z), n \geqq N$, it follows that in or on the curve $G\left(r_{k}+\epsilon, r+\epsilon ; p-k, \alpha_{k}\right)$ lie exactly $p$ zeros of $f(z)$. Since $\epsilon$ is an arbitrary, small positive number, it follows that exactly $p$ zeros of $f(z)$ lie in or on the curve $G\left(r_{k}, r ; p-k, \alpha_{p}\right)$ as stated in Theorem (4.2).

University of Wisconsin, MilwaukeE 